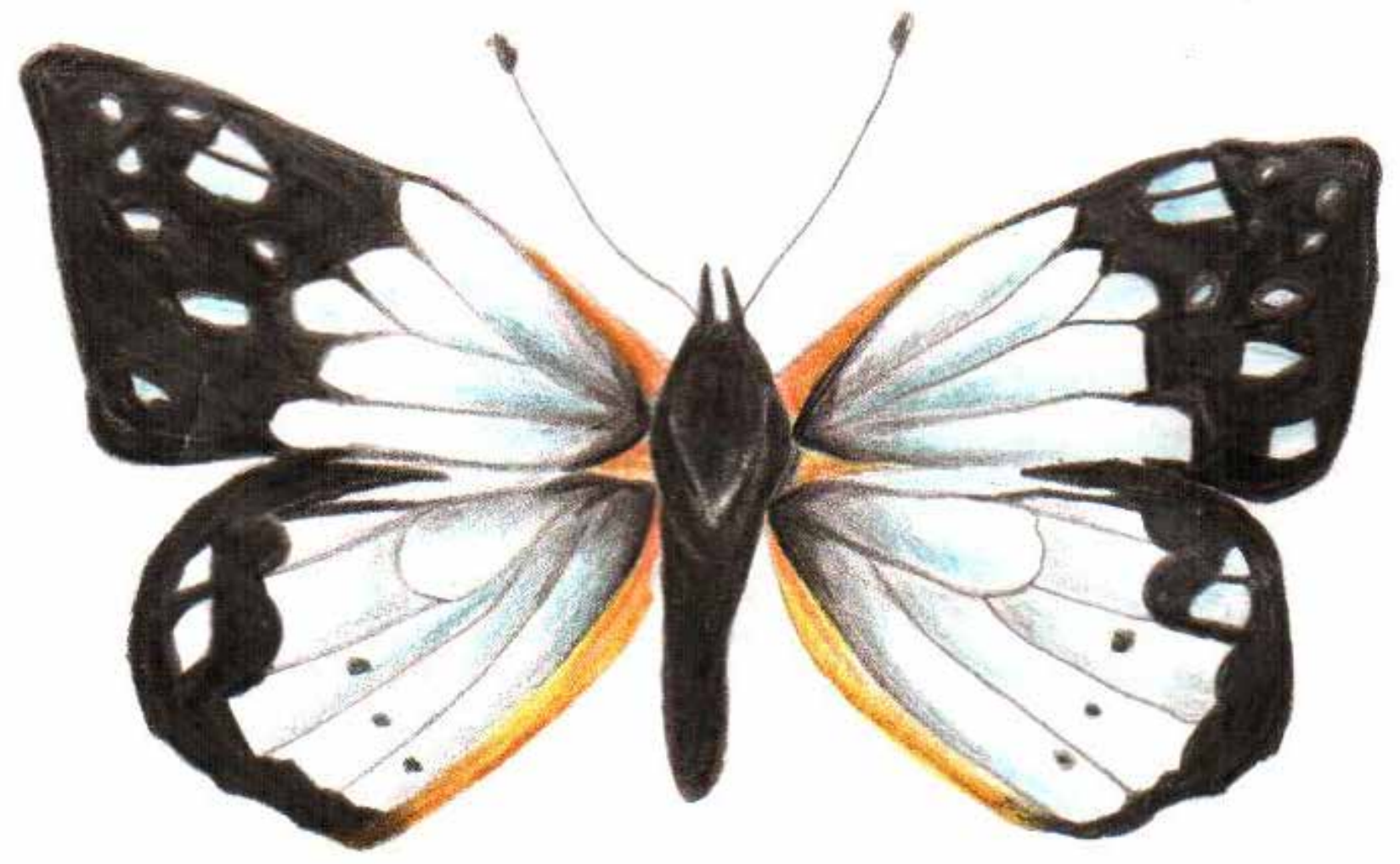

Jeny Andrea Fuentes Acevedo

Estudiante de Sexto Semestre de Licenciatura en Biología

Universidad Pedagógica Nacional

Técnica: Lápices de colores 


\section{LOS MARIPOSARIOS COMO ESPACIOS QUE POTENCIAN Y ENRIQUECEN LA ENSEÑANZA DE LAS CIENCIAS}

Fecha de recepción: 24 de marzo de 2014 Fecha de aprobación: 15 de junio de 2014

\author{
Gina Marcela Robayo Vélez ${ }^{1}$
}

\section{Resumen}

Este trabajo es el resultado de la práctica pedagógica intensiva llevada a cabo en la IED Orlando Fals Borda con los estudiantes del club de ciencias Tataxue y con los estudiantes del grado 702 de la jornada tarde, con el objetivo de apoyar e implementar actividades en el uso del mariposario de la institución que puedan potenciar y enriquecer la enseñanza de las ciencias. Con el método de investigación cualitativo y el ciclo de indagación propuesto por la guía metodológica de la Enseñanza de la Ecología en el Patio de la Escuela (EEPE), se realizaron actividades de crecimiento y desarrollo, guías de apoyo, laboratorios, observaciones, presentaciones, pretest y postest, entrevistas, etc. Después de la implementación se obtuvo que los mariposarios son espacios de observación, exploración, experimentación e investigación que involucran tanto aspectos investigativos como también humanos, que ofrecen un lugar alterno e interactivo que intenta atraer o seducir al estudiante y mantenerlo con una serie de posibilidades que están sujetas a la imaginación y a las expectativas de quien lo desarrolle. Además puede abarcar no solo al estudiante sino también gran parte de su núcleo familiar y educativo, lo que enriquece y contextualiza mucho más la experiencia de aprender y de enseñar.

\section{Palabras clave}

Ciclo de indagación de primera mano, Mariposario, método de investigación cualitativo, enseñanza de las ciencias.

\section{Abstract}

This work is the result of the intensive pedagogical practice conduced at the Orlando Fals Borda school I.E.D. (Educational Institution District), with the Taxue`s science club students and the 702 student's, with the aim of supporting and implementing activities in the use of butterfly of the institution that can enhance and enrich the teaching of science. Using qualitative research method and the inquiry cycle proposed for the methodological guide the Teaching of Ecology in the Yard School. Activities were made of growth and development, support guides, labs, observations, presentations, pre-test and post-test, interviews, etc. After implementation it was found that the butterfly farm are spaces for observation, exploration, experimentation, and research involving not only investigative but also human aspects that provide and alternative and interactive site than trying to attract or seduce the student and keep a series of possibilities that are subject to the imagination and expectations of who develop it, also can encompass not only student, but also a large part of their family and educative. Enriching and contextualizes much more the experience of learning and teaching.

\section{Key words}

Inquiry cycle firsthand, Butterfly farm, Qualitative research method, Science teaching.

1 Estudiante de Licenciatura en Biología de la Universidad Pedagógica Nacional. Trabajo de práctica pedagógica integral, 2013. Correo electrónico: ginarob.20@gmail.com 


\section{Introducción}

Como parte de la formación de licenciados en biología, las prácticas pedagógicas tienen gran importancia en la conformación de la identidad del ser maestro. Por consiguiente, este proyecto representó una experiencia formativa importante, ya que permitió integrar los saberes biológicos acerca de los insectos-específicamente de las mariposas-, y posibilitó el acercamiento a los contextos educativos, donde se articulan las expectativas del aprendizaje, los contenidos a enseñar, las concepciones de los estudiantes y las actividades planteadas; de modo que el abordaje de los contenidos y el quehacer en el aula constituyeron un proceso dinámico y progresivo tanto para el estudiante, como para el profesor.

El objetivo de la propuesta fue enriquecer la enseñanza de la ciencias por medio del uso del mariposario de la institución, de manera que proporcionara experiencias diferentes al aula de clase, desde dinámicas de trabajo y objetivos planteados diferentes a los que se desarrollaría en una sesión, hasta reflexiones sobre características más específicas sobre mantenimiento de los ecosistemas, conservación de las especies, importancia de las variables climáticas en la supervivencia de estos organismos, hasta diseño de tecnologías a partir del conocimientos en diferentes áreas que complementan y enriquecen la enseñanza de las ciencias

La razón para escoger las mariposas y en general los insectos se debió a que estos organismos representan

[...] un material estimulante en el desarrollo de la curiosidad de los estudiantes, así mismo sus características biológicas permiten un acercamiento a la comprensión de la vida y todas sus manifestaciones debido a que su ciclo de vida es relativamente corto y su cambio físico es dramático durante su desarrollo... (Torres, 2004 pp. 512).

Además, son relativamente fáciles de obtener y manipular para la enseñanza de los programas académicos de los estudiantes.

Por otro lado, el trabajo estuvo desarrollado bajo el método cualitativo, ya que permite reconocer la existencia de múltiples realidades, además admite la apertura a la obtención de resultados inesperados que no utiliza categorías preestablecidas por lo que la información obtenida puede ser más representativa de la experiencia de los estudiantes sobre lo realizado en el mariposario. Algunas de las actividades realizadas en este trabajo, están planteadas bajo la propuesta del ciclo de indagación de la Guía Metodológica de Enseñanza de la Ecología en el Patio de la Escuela
(EEPE) (2002), debido a que en esta guía, se parte de la cotidianidad del estudiante, como por ejemplo el patio de la institución (sitios comunes, conocidos y frecuentados por los estudiantes) y se utilizan como escenarios prácticos (cercanos, familiares) de enseñanza en donde se "aprende haciendo yse aprende reflexionando"(Arango, Chaves y Feisinger, 2002, p xv) , aquí “...los docentes y sus estudiantes construyen preguntas sobre su entorno (natural, social, cultural) y las responden a través de la acción" (Arango, Chaves y Feinsinger, 2002, p xv).

Como resultado, los estudiantes desarrollaron su propia investigación inspirada en el trabajo con las mariposas y el estudio de las escamas y los paneles solares. Para ello se implementaron una serie de guías y sesiones acerca de los insectos y las mariposas, acompañadas de laboratorios, consulta de información y cuidado y seguimiento del ciclo de vida de las mariposas "Monarca" (Danaus plexippus) y "amarilla" (Phoebis sennae).

Por último, con este proyecto se pretendió proporcionar un espacio que permitiera la reflexión y el uso de habilidades y conocimientos aplicables en la cotidianidad y que contribuyeran a la formación de un pensamiento crítico y reflexivo acerca de las problemáticas ambientales globales que en un país como el nuestro tiene un impacto considerable sobre ecosistemas y zonas que son vitales para la supervivencia de las especies (incluyéndonos).

\section{Situación Problema}

Esta iniciativa se plantea a partir del interés de la IED Orlando Fals Borda por poner de nuevo en funcionamiento el mariposario Panambi que había sido construido en años anteriores, con el objetivo de aprovecharlo como espacio educativo.

Por consiguiente, se propone utilizar espacios diferentes al aula de clase que sean atrayentes para los estudiantes y que además enriquezcan su procesos de aprendizaje de las ciencias; para lo cual se involucran también diferentes actores de la institución, no solo estudiantes y profesores, de manera que el mariposario sea un puente entre las diferentes temáticas planteadas en las áreas de biología y ciencias de algunos cursos, y con la metamorfosis, el papel ecológico de estos y otros organismos en el ambiente, las relaciones biológicas que mantiene con su entorno, etc., temas de corte ambiental y ecológico; además de abordar la protección y conservación de organismos.

Esta experiencia aborda situaciones ambientales de vital importancia que requieren de una reflexión y de la toma de decisiones por parte del público en general y, en especial, de la comunidad del colegio. Igualmente, el uso de 
espacios como este puede ayudar a mostrar diferentes perspectivas que lleven a soluciones responsables.

Para dar respuesta a esta situación se plantearon los siguientes objetivos:

- Apoyar e implementar actividades en el uso del mariposario de la IED Orlando Fals Borda como un espacio educativo para la enseñanza de las ciencias.

- Caracterizar las nociones de los estudiantes sobre los insectos y las mariposas.

- Evidenciar el papel ecológico y la importancia de las mariposas en el ambiente a través de la experiencia de crecimiento y desarrollo de mariposas.

- Diseñar actividades académicas que promuevan conocimientos ecológicos y de conservación de las mariposas y otros organismos.

- Diseñar actividades que promuevan habilidades investigativas en los estudiantes.

\section{Contextualización}

El proyecto se llevó a cabo en la IED Orlando Fals Borda ubicada en la localidad de Usme, en el barrio Santa Librada, de Bogotá, con estudiantes de la jornada tarde del club de astronomía y ciencias Tataxue, que se encuentran entre los 10 y 20 años, la mayoría de ellos hombres. Además, se desarrollaron algunas actividades con estudiantes del grado 702, en edades entre los 11 y 14 años.

El club Tataxue ("hijos de las estrellas" en muisca), es un

[...] grupo de estudiantes, docentes y padres de familia comprometidos con el desarrollo de valores y actitudes ciudadanas, que pretende incentivar la alfabetización científica y tecnológica con el fin de resolver problemas locales en el marco de la filosofía falsbordiana de lograr la emancipación de la sociedad. Para cumplir con este fin, se pretende formar líderes en temas afines con la astronomía, la ciencia y la tecnología, logrando articular la dimensión ambiental al desarrollo humano integral. (IED Orlando Fals Borda, 2010).

Por otra parte, los integrantes del club tienen la posibilidad de escoger entre cuatro líneas de investigación: Astronomía, Astrobiología, Reutilizarte y Biofilia. En Biofilia, que es la línea en la que se adelantó el proyecto, principalmente se ha trabajado sobre los efectos del cambio climático en los ciclos biológicos de insectos, en especial de Leptophobia aripa (mariposa blanca). Igualmente el club ha publicado un libro titulado: Ciudadanos ante el cambio climático, una mirada de la realidad ambiental desde la perspectiva astronómica. Además, ha participado en múltiples ferias de ciencias y astronomía.

\section{Fundamento Disciplinar}

\section{¿Qué es una Mariposa?}

Como lo mencionan Gullan y Cranston (2010), las mariposas son insectos pertenecientes al orden de los lepidópteros (alas con escamas) entre los que también se incluyen las polillas y los skippers o Capitanes (Mariposas de la familia Hesperiidae). El cuerpo de las mariposas como el de todos los insectos está dividido en tres partes o tagmas: cabeza, tórax y abdomen. Su desarrollo es holometábolo (metamorfosis completa).

La cabeza es hipognata (hacia abajo), y porta una larga probóscide enrollada, que se desenrolla cuando la mariposa va a alimentarse, En la cabeza se encuentran un par de ojos compuestos, formados por multitud de facetas independientes, que proporcionan cierta visión y que son sensibles a distintas longitudes de onda. Los ojos compuestos son grandes, y los ocelos (órganos sensoriales pareados ubicados dorsolateralmente sobre la cabeza) son frecuentes. Las antenas son mutisegmentadas, por lo general clavadas (en forma de maza).

El protórax es pequeño, con un par de placas ubicadas dorsolateralmente, mientras que el mesotórax es grande y posee escuto y escutelo. El metatórax es pequeño. Las alas están completamente cubiertas por una doble capa de escamas (macrotrichias modificadas y aplanadas), y las alas anteriores y posteriores están acopladas mediante un frénulo, un yugo o simple superposición. La venación alar está constituida de venas longitudinales, unas pocas transversales y algunas celdas grandes como la celda discal que es la más grande. Las patas son largas y corredoras, con cinco tarsómeros. El abdomen posee 10 segmentos, con un segmento variablemente reducido, y los segmentos 9 y 10 modificados como una armadura genital externa. Los genitales internos de la hembra son bastante complejos.

Las mariposas llevan a cabo una serie de cambios extraordinarios en su vida, los cuales se dividen en cuatro etapas: huevo, oruga (larva), crisálida (pupa), adulto (imago).

Mariposario: es una construcción encerrada en malla y acondicionada, con humedad, temperatura y alimentación adecuadas para la crianza y la exhibición de mariposas en su medio natural. 
Condiciones del mariposario: para las condiciones del mariposario se seguirán algunas sugerencias del texto Plan de manejo, del CENTRO DE MANEJO DE VIDA SILVESTRE AWACACHI.(2006) El mariposario estará rodeado por una malla tipo mosquitero para impedir la entrada de aves u otros depredadores, es importante que esté ubicado en un lugar donde no corra muy fuerte el viento, ya que esto puede lastimar a las mariposas, para ello se ubicarán alrededor de las paredes plantas altas que puedan ayudar a contener las corrientes de aire. Es recomendable que tenga una forma rectangular para que las mariposas estén más cómodas, además debe tener suficiente sombra para evitar su deshidratación.

Mantenimiento de las larvas: se mantendrán en recipientes de plástico transparente con tapa (tipo almuerzo o baso), la tapa tendrá una rejilla de tela de mosquitero para que circule el aire. Adentro del recipiente se colocarán hojas de la planta nutricia que se cortarán y se mantendrán con un poco de papel higiénico o algodón mojado para que la hoja esté lo más fresca posible hasta que la larva la haya consumido.

Cuando la larva se haya colgado y empiece su transformación a pupa, se tomará la hoja de la que se colgó, o la tapa del recipiente si se colgó en esta, y se llevarán al mariposario, para que en caso de que salga no quede encerrada en el recipiente.

Para la alimentación de los adultos se tendrán esponjillas embebidas en miel de abejas, y también se tendrán platos de fruta de patilla, piña guayaba o papaya, además de plantas con flores que produzcan néctar adecuado para ellas.

Colecta: para la obtención de los organismos, lo primero que se debe hacer es identificar las plantas hospederas y la colecta de los huevos, aquí se revisa cuidadosamente la planta y una vez ubicado un huevo en una hoja o rama se procede a cortarla; luego se debe tratar de mantener la hoja lo más fresca posible con algodón o papel higiénico mojado en agua. Posteriormente se traslada la hoja con el huevo a un recipiente de plástico como los anteriores mencionados y se deja allí, asegurándose de que el algodón no se seque.

Para la colecta de los adultos, se utilizará una jama, que es una red especial para capturar estos animales sin dañarlos. El mango de la red debe ser liviano y fuerte aproximadamente $80 \mathrm{~cm}$, el aro debe tener $37,5 \mathrm{~cm}$ y estar hecho de un alambre dulce grueso calibre 5 . El material de la bolsa debe ser tela tul y la bolsa debe estar elaborada de una sola pieza cocida con la costura francesa.
Una vez capturados, los adultos se dispondrán en unos sobres de papel Bond para su trasporte hasta llegar al mariposario, como lo recomiendan Borror y Dwight (1970). Estos sobres se hacen tomando una hoja de papel y luego se dobla una esquina sobre la otra, de manera que forme un triángulo con dos pestañas grandes; luego se toma cada pestaña y se dobla una para cada lado, cosa que no quede ningún hueco por el que se pueda escapar la mariposa, después del último plegado, quedan dos pestañitas en forma de triángulo que se doblan para asegurar el sobre.

\section{Fundamento Pedagógico}

\section{Enseñanza de la Ecología en el patio de la escuela}

\section{Ciclo de Indagación.}

El ciclo de indagación es una propuesta de enseñanza que se vale de espacios cercanos y comunes o conocidos por los estudiantes, como por ejemplo, las zonas verdes, los patios o cualquier zona de fácil acceso, y los utiliza como escenarios prácticos de enseñanza, en donde se "aprende haciendo y se aprende reflexionando. [Aquí] los docentes y sus estudiantes construyen preguntas sobre su entorno (natural, social, cultural) y las responden a través de la acción" (Arango, Chaves y Feisinger, 2002 ).

Para lograr este propósito la guía recomienda lo siguiente:

a. Se selecciona un lugar dentro del colegio y, si se puede, se delimita; éste espacio debe ser tener ciertas características que permitan que los estudiantes se planteen preguntas susceptibles de responder por medio de una investigación realizada por ellos mismos; por consiguiente, el lugar debe ser atractivo o interesante, en este caso, el espacio delimitado fue el mariposario, que se ubicó dentro de la institución cerca a la entrada principal.

b. La guía propone el planteamiento de unas preguntas de investigación por parte de los estudiantes a partir de la observación e interacción dentro del lugar; para propósitos del trabajo, la indagación fue semiguiada, lo que quiere decir que el investigador propuso un tema global, en este caso las mariposas, y la intervención fue solo para asegurar que las preguntas planteadas estuvieran orientadas bajo la EEPE, es decir, que fueran factibles de ser resueltas en un periodo razonable, además de comparativas, interesantes y comprensibles. 
c. Luego de tener una pregunta con las características anteriormente mencionadas, se pasa a la etapa de la acción o la experiencia de primera mano, en donde, el investigador recoge la información para responder a su pregunta; esta etapa le permite al estudiante organizar sus objetivos, diseñar las estrategias e instrumentos para la recolección de datos.

d. Una vez colectados los datos, viene la etapa en la que el estudiante puede reflexionar sobre si lo obtenido respondió su pregunta, si no, indagar sobre las posibles causas, si le surgieron más preguntas, y lo que puede concluir de ello.

\section{Metodología}

\section{Método cualitativo de Investigación}

El desarrollo del proyecto estuvo orientado con un enfoque de investigación cualitativo, el cual

[...] trata de identificar la naturaleza profunda de las realidades, su estructura dinámica, aquella que da razón plena de su comportamiento y manifestaciones. De aquí, que lo cualitativo (que es el todo integrado) no se opone a lo cuantitativo (que es soilo un aspecto), sino que lo implica e integra, especialmente donde sea importante. (Martínez, s.f.).

Se optó por este enfoque, ya que lo que se pretendió fue presentar el mariposario como un espacio alterno de enseñanza que fuera atractivo e interactivo con los estudiantes, para ello fue necesario llevar a cabo una evaluación constante de la efectividad del espacio, de las actividades dentro de este, del interés que se percibía por parte de los estudiantes. Por consiguiente se implementaron: un pretest y postest, las guías construidas, el ejercicio de crecimiento y desarrollo, la entrevista semiestructurada y la observación no participativa. Todo lo anterior estuvo sujeto a interpretaciones y reflexiones a lo largo de toda la práctica.

El trabajo se llevó a cabo en varias etapas:

1. Diagnóstico acerca de la temática general, por medio de la utilización del instrumento N. ${ }^{\circ} 1$.

2. Desarrollo de las actividades propuestas en el mariposario.

- Propuestas del ejercicio de crecimiento y desarrollo. Instrumento N. ${ }^{\circ} 2$.

- Guías de trabajo, actividades de cada sesión. Instrumento N. ${ }^{\circ} 3$.
3. Recolección de información, guías de trabajo, observación (fotografías); realización de entrevistas y postest.

4. Análisis de la información de los instrumentos anteriormente mencionados.

\section{Muestra Intencional}

- Para ello se escogió el Colegio Orlando Fals Borda IED, y los miembros del club de ciencias Tataxue, específicamente los estudiantes de la línea de Biofilia pertenecientes a los grados décimo, octavo, séptimo y sexto; sin embargo con los demás integrantes del club se trabajaron aspectos más generales.

- Por otro lado se trabajó con los estudiantes del grado 702, debido a un interés manifestado por los mismos alumnos, de abordar temas relacionados con el reconocimiento de los insectos en general y con las mariposas.

\section{Instrumentos Utilizados}

\section{Instrumento $N^{\circ} 1$.}

La implementación de un pretest y un postest (ver anexos) con el fin de averiguar inicialmente qué ideas o conceptos tenían los estudiantes alrededor de las mariposas y del trabajo con ellas, por ello, las respuestas se categorizaran según aspectos comunes en ellas como por ejemplo: categoría (aspectos morfológicos), así, al finalizar, poder hacer una comparación con los resultados del postest para determinar si hubo algún cambio. Tanto el pretest como el postest se aplicaron a los integrantes de biofilia y a 14 estudiantes de grado séptimo, cabe aclarar que el pretest y el postest contuvieron las mismas preguntas a excepción de la última pregunta.

\section{Instrumento № 2.}

En el ejercicio de crecimiento y desarrollo se les proporcionó organismos vivos de Danausplexippus (Monarca) y Phoebissennae (mariposa amarilla), en diferentes etapas (huevos, orugas, pupas, adultos), para que en una tabla de seguimiento llevaran el registro durante la semana del ciclo de vida, al igual que un registro fotográfico; además, los estudiantes debían realizar observaciones al estereoscopio de las diferentes etapas. Una parte importante es que los estudiantes del club recibieron a sus compañeros y a estudiantes de otros cursos para explicar e instruir sobre el mariposario y su mantenimiento. El seguimiento se llevó a cabo durante dos meses y medio en el mariposario Panambi. Por otra parte se tuvieron en cuenta características como temperatura y humedad. 
Instrumento $\mathbf{N} \bullet \mathbf{3}$.

Guías construidas: las guías se realizaron con el propósito de posibilitar al estudiante la interacción directa con los insectos a trabajar; además proponen una observación constante de los mismos, con actividades como observaciones, dibujos, búsquedas, fabricación de materiales de colecta, observación bajo el estereoscopio y preguntas para indagar en Internet, entre otras, que buscan mantener la atención y la motivación del estudiante, y desarrollar en este habilidades investigativas.

\section{Instrumento № 4.}

La observación: permite al investigador conocer directamente el contexto en el que se desarrollan los individuos $y$, por consiguiente, acceder al conocimiento a partir de un registro de las acciones de las personas en su ambiente cotidiano. Para dirigir la observación de las actividades realizadas con los estudiantes la información será guiada por las siguientes preguntas sugeridas por Elssy Bonilla (1997). ¿Quién es?, ¿qué hace?, ¿con quién?, ¿en qué situación?, ¿dónde?, ¿qué tecnología y que artefactos se usan? ¿durante cuánto tiempo?, ¿qué están tratando de lograr?, ¿qué emociones y sentimientos se están expresando?, que serán evidenciadas en el análisis de los resultados de las actividades.

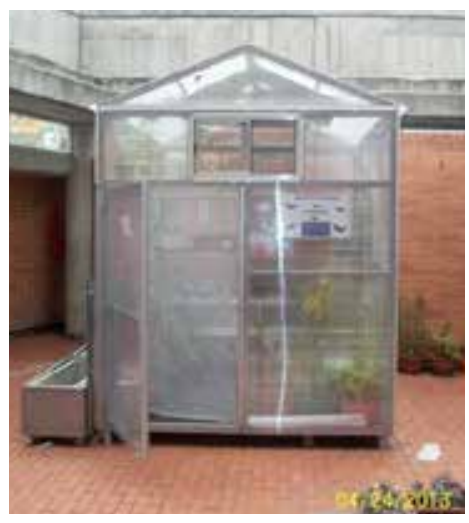

Imagen 1. Mariposario Panambi Tomado por: (C)ina Robayo.

\section{Instrumento $\mathrm{N}^{\circ} 5$.}

Entrevista semiestructurada: se optó por esta, ya que aunque cuenta con un tema o preguntas preestablecidas, el sujeto entrevistado tiene mayor libertad para informar sobre el tema que el entrevistador define, sin que ello llegue a ejercer un dominio total sobre el informante, así el entrevistador mantiene la conversación enfocada en un tema particular y le proporciona al informante el espacio y la libertad suficientes para definir el contenido de la discusión. La entrevista semiestructurada se realizó al profesor tutor, quien también es el director del club Tataxue, una vez finalizada la práctica.

Para el análisis de la información se pretendió hacer un contraste de los instrumentos, es decir, buscando cómo se combinaban y se diferenciaban los resultados, esto significa, que se observó el contenido central de los mismos para determinar qué es significativo y a partir de allí, reconocer patrones y categorías de esos datos y así relacionarlos entre sí, para elaborar una explicación integrada. Se tomaron en cuenta las categorías surgidas del pretest y postest, el desempeño de los estudiantes en la realización de las actividades, los comentarios del titular y, en espacial, el proyecto planteado por los estudiantes, producto del trabajo de la práctica.

\section{Resultados y Discusión}

\section{Pretest y Postest.}

Se puede evidenciar que los estudiantes tienen un mayor reconocimiento de las características de las mariposas (tabla 2), ya que argumentan desde nombres propios, hasta particularidades de los insectos, relacionando esa información (morfológica y biológica) de las mariposas, para responder a sus preguntas; en contraste con sus respuestas del pre-test (tabla $n^{\circ} 1$ ) en la que los estudiantes se fijaron más en describir características estéticas y en agrupar a las mariposas sin mayores descripciones y explicaciones. Un aspecto importante, está relacionado con la mención de organismos como bioindicadores (tablas 1 y 2), que reflejan un reconocimiento de las interacciones de estos organismos con su ambiente, en parte debido a sus trabajos anteriores en el club. 
Para propósitos de organización de la información, se usó un código de identificación en las tablas: Est de ESTUDIANTE, acompañado de la letra $\mathrm{N}^{\circ}$ y un número del 1 al 17, que hace referencia a la información de ese estudiante en su pretest o postest.

\begin{tabular}{|c|c|c|c|}
\hline Pregunta & Categoría & $\begin{array}{c}\text { N.o de } \\
\text { est. }\end{array}$ & Evidencia o comentarios \\
\hline \multirow[t]{5}{*}{ ¿Qué es una mariposa? } & Asociado a su uso como bioindicadores. & 3 & Est. N. ${ }^{\circ}$ 2: Es un animal llamativo y bioindicador. \\
\hline & Referente a sus hábitos y morfología. & 5 & $\begin{array}{l}\text { Comentarios: polinizador. } \\
\text { Se alimenta de polen } \\
\text { Tiene alas. }\end{array}$ \\
\hline & $\begin{array}{l}\text { Asociado al grupo al que pertenecen. } \\
\text { Insectos. }\end{array}$ & 7 & $\begin{array}{l}\text { Est. N. }{ }^{\circ} \text { : La mariposa es un insecto muy importante. } \\
\text { Est. N. }{ }^{\circ} \text { 12: Es un animal en forma de insecto. }\end{array}$ \\
\hline & Referente a su aspecto. & 1 & $\begin{array}{l}\text { Est. N. }{ }^{\circ} \text { 13: Es aquello que conocemos como una animal } \\
\text { muy raro. }\end{array}$ \\
\hline & No responde. & 1 & \\
\hline
\end{tabular}

Tabla 2. Pregunta 1 del postest

\begin{tabular}{|c|c|c|c|}
\hline Pregunta & Categoría & $\begin{array}{l}\text { N. }{ }^{\circ} \text { de } \\
\text { est. }\end{array}$ & Evidencia o comentarios \\
\hline \multirow[t]{4}{*}{ ¿Qué es una mariposa? } & $\begin{array}{l}\text { Identifican el grupo al que pertenecen con } \\
\text { mayor profundización. }\end{array}$ & 4 & $\begin{array}{l}\text { Est. N. }{ }^{4} \text { : Las mariposas son insectos que pertenecen a los } \\
\text { lepidópteros (bioindicadores). }\end{array}$ \\
\hline & $\begin{array}{l}\text { Referente a las características de los } \\
\text { insectos. }\end{array}$ & 7 & $\begin{array}{l}\text { Est. N. }{ }^{\circ} 11: \text { Es un insecto que tiene seis patas y tiene tres } \\
\text { partes. }\end{array}$ \\
\hline & Asociado a los hábitos y estructuras. & 4 & $\begin{array}{l}\text { Est. N. }{ }^{\circ} \text { 6: Es un insecto volador, que come polen con la } \\
\text { espiritrompa. }\end{array}$ \\
\hline & No responden. & 2 & \\
\hline
\end{tabular}

En cuanto a los dibujos del cuerpo de los organismos, inicialmente hay mayor inclinación a dibujar características antropomórficas (características humanas) (tabla 3) como sonrisas, zapatos y corazones en las alas, debido a que estas nociones se encuentran en la cotidianidad del estudiante, las experiencias, el lenguaje común y en el medio social. Esto no quiere decir que los dibujos sean incorrectos, sino que son la representación que los estudiantes han construido acerca de este organismo según su experiencia hasta ese momento. Una vez desarrollado el proyecto, hay una mayor tendencia a dibujar estructuras específicas de las mariposas como los ojos o la espiritrompa, ya que se hicieron observaciones al estereoscopio de estos organismos, además de las experiencias en el mariposario en donde muchos tuvieron la oportunidad de alimentarlos de su propia mano. 
Tabla 3. Pregunta 2 pretest

\begin{tabular}{|c|c|c|c|}
\hline Pregunta & Categoría & $\begin{array}{l}\text { N. }{ }^{\circ} \text { de } \\
\text { est. }\end{array}$ & Evidencia o comentarios \\
\hline \multirow{5}{*}{$\begin{array}{l}\text { ¿Cómo es el cuerpo de una } \\
\text { mariposa? Dibújalo en la } \\
\text { parte de atrás }\end{array}$} & $\begin{array}{l}\text { Comparación con un objeto, sin deta- } \\
\text { lles. }\end{array}$ & 2 & Est. N. ${ }^{\circ}$ : El cuerpo es similar a un palo grueso. \\
\hline & Morfología detallada. & 3 & $\begin{array}{l}\text { Comentario: dibujos con patrones de diseño, color y forma } \\
\text { en las alas y aparato bucal. }\end{array}$ \\
\hline & $\begin{array}{l}\text { Características antropomórficas y } \\
\text { caricaturescas. }\end{array}$ & 4 & $\begin{array}{l}\text { Comentario: alas con corazones. } \\
\text { Ojos y pico de aves. } \\
\text { Sin patas o con zapatos. }\end{array}$ \\
\hline & Sin mayor detalle. & 7 & $\begin{array}{l}\text { Comentario: muestra la silueta de las mariposas, sin } \\
\text { embargo no hay detalle en características particulares de las } \\
\text { mismas. }\end{array}$ \\
\hline & No dibuja. & 1 & \\
\hline
\end{tabular}

Tabla 4. Pregunta 2 postest

\begin{tabular}{|c|c|c|c|}
\hline Pregunta & Categoría & $\begin{array}{l}\text { N. }{ }^{\circ} \text { de } \\
\text { est. }\end{array}$ & Evidencia o comentarios \\
\hline \multirow[t]{5}{*}{$\begin{array}{l}\text { ¿Cómo son las partes del } \\
\text { cuerpo? }\end{array}$} & Morfología de cada parte detallada. & 8 & $\begin{array}{l}\text { Est. N. } 10 \text { : Las mariposas tienen cabeza, tórax abdomen, ante- } \\
\text { nas, partes de la boca espiral, ojos con hexágonos, y seis patas. }\end{array}$ \\
\hline & Características antropomórficas. & 3 & Comentario: corazones \\
\hline & Sin detalle. & 3 & \\
\hline & Comparación con objetos. & 2 & Comentario: similares a cilindros o palos \\
\hline & No responde. & 1 & \\
\hline
\end{tabular}

Por otro lado, hay un evidente avance en cuanto al reconocimiento del ciclo de vida de las mariposas (tabla 6, est. N. ${ }^{5}$ ), la mayoría identifica y conoce alguna información respecto a las etapas de la metamorfosis, y empiezan a relacionarlas con otros organismos con ciclos de vida similares, sin embargo, la importancia de este proceso en relación con la interacción con otros organismos, la supervivencia, y en general con el ambiente aún no es clara. Sin embargo, la experiencia de ver la transformación de este organismo es muy atractiva para los estudiantes; muchos de ellos en un inicio pensaron que cada etapa era un animal diferente especialmente la oruga, además, fue fuente principal de sus inquietudes y preguntas. 
Tabla 5. Pregunta 3 pretest

\begin{tabular}{|c|c|c|c|}
\hline Pregunta & Categoría & $\begin{array}{l}\text { N. }{ }^{\circ} \text { de } \\
\text { est. }\end{array}$ & Evidencia o comentarios \\
\hline \multirow[t]{4}{*}{$\begin{array}{l}\text { ¿Qué sabes acerca de su } \\
\text { ciclo de vida? }\end{array}$} & Referente a las etapas de la metamorfosis. & 10 & \\
\hline & Referente al tiempo de vida. & 3 & $\begin{array}{l}\text { Est. N. }{ }^{\circ} \text { : Yo sé de su ciclo de vida que hay unas mari- } \\
\text { posas que duran un día, dos meses, un año. }\end{array}$ \\
\hline & $\begin{array}{l}\text { Confusiones con respecto a las etapas de } \\
\text { la metamorfosis. }\end{array}$ & 3 & $\begin{array}{l}\text { Est. N. 3: Que hay algunas que se saltan su ciclo de } \\
\text { vida normal y otras que se saltan, huevo, larva, pupa, } \\
\text { adulto. }\end{array}$ \\
\hline & No responde. & 1 & \\
\hline
\end{tabular}

Tabla 6. Pregunta 3 postest

\begin{tabular}{|c|c|c|c|}
\hline Pregunta & Categoría & $\begin{array}{l}\text { N. }{ }^{\circ} \text { de } \\
\text { est. }\end{array}$ & Evidencia o comentarios \\
\hline $\begin{array}{l}\text { ¿Qué sabes acerca de su } \\
\text { ciclo de vida? }\end{array}$ & $\begin{array}{l}\text { Referente a las cuatro etapas de la } \\
\text { metamorfosis. }\end{array}$ & 13 & $\begin{array}{l}\text { Est. N. }{ }^{5} \text { : Primero es huevo, después pasa a ser una larva y } \\
\text { para después volverse pupa o crisálida y después pasa a ser } \\
\text { mariposa adulta y ahí se termina el ciclo de ella. }\end{array}$ \\
\hline & $\begin{array}{l}\text { Confusión de las etapas de la metamor- } \\
\text { fosis. }\end{array}$ & 2 & Est. N. ${ }^{\circ} 12:$ huevo, larva, oruga, mariposa \\
\hline & Referente a tiempo. & 1 & \\
\hline & No responde. & 1 & \\
\hline
\end{tabular}

Por otro lado, se evidencia un acercamiento a temáticas como la defensa, ya que algunos identificaron estrategias como el camuflaje, y los colores de advertencia de la familia Monarca (Danaus plexippus), además del tipo de recursos que puede explotar un organismo, ya que muchas de sus preguntas estaban relacionadas con, por qué las mariposas que se criaron unas comían de una sola planta y las otras podían comer de dos. También observaron el vuelo y notaron que, dependiendo de la especie, en unas es más armonioso que en otras. 
Tabla 7. Pregunta 4 pretest

\begin{tabular}{|c|c|c|c|}
\hline Pregunta & Categoría & $\begin{array}{c}\text { N. }{ }^{\circ} \text { de } \\
\text { est. }\end{array}$ & Evidencia o comentarios \\
\hline \multirow[t]{5}{*}{ ¿Qué las has visto hacer? } & Alimentación & 3 & Est. N. ${ }^{\circ}$ 15: Tomar el néctar de las flores. \\
\hline & Referente al vuelo. & 10 & \\
\hline & Referente a la reproducción. & 1 & Est. N. ${ }^{\circ}$ 13: Poner huevos. \\
\hline & Referente al estado de reposo. & 1 & Est. N. ${ }^{\circ}$ : Sentarsen en una planta. \\
\hline & No desarrolla. & 2 & \\
\hline
\end{tabular}

Tabla 8. Pregunta 4 postest

\begin{tabular}{|c|c|c|c|}
\hline Pregunta & Categoría & $\begin{array}{l}\text { N. }{ }^{\circ} \text { de } \\
\text { est. }\end{array}$ & Evidencia o comentarios \\
\hline ¿Qué las has visto hacer? & Referente a la alimentación. & 6 & \\
\hline & Referente al vuelo. & 7 & \\
\hline & Asociado a su capacidad de camuflaje. & 3 & $\begin{array}{l}\text { Est. N. }{ }^{\circ} \text { 13: La mariposa se escondió en una hoja y se } \\
\text { parecía a una hoja. }\end{array}$ \\
\hline & No responde. & 1 & \\
\hline
\end{tabular}

Se observa una prevalencia del valor estético. Como se muestra en las tablas 9 y 10 , el estudiante menciona que sus familiares dicen "que son muy hermosas" (est. N. $\left.{ }^{\circ} 15\right)$, afinidad que puede ser el anclaje para trabajar diferentes temas en ciencias que no sean muy claros por el público en general. Sin embargo, no hay ningún comentario relacionado con su importancia en el mantenimiento de los ecosistemas o creencias alrededor de las mariposas, que era la intención de esta pregunta. 
Tabla 9. Pregunta 5 pretest

\begin{tabular}{|c|c|c|c|}
\hline Pregunta & Categoría & $\begin{array}{l}\text { N. }{ }^{\circ} \text { de } \\
\text { est. }\end{array}$ & Evidencia o comentarios \\
\hline \multirow{8}{*}{$\begin{array}{l}\text { ¿Qué has escuchado de amigos } \\
\text { familiareso vecinos acerca de ellas? }\end{array}$} & Asociado con la apariencia. & 7 & Est. N. ${ }^{\circ}$ 15: Que son muy hermosas. \\
\hline & Asociado con las plantas hospederas. & 1 & Est. № 5: Que una mata el ganado. \\
\hline & Asociado con bioindicadores. & 1 & \\
\hline & Referente a mantenerlas cautivas. & 1 & $\begin{array}{l}\text { Est N. }{ }^{\circ} \text { : Ps que son chebres y que les gustaría cazar- } \\
\text { las. }\end{array}$ \\
\hline & Asociado con el peligro. & 1 & Est N. ${ }^{\circ} 12$ : Que no son mariposas muy peligrosas. \\
\hline & Referente a la diversidad. & 1 & \\
\hline & Asociado al tiempo de vida. & 2 & \\
\hline & No responde. & 3 & \\
\hline
\end{tabular}

Tabla 10. Pregunta 5 postest

\begin{tabular}{|l|l|l|l|}
\hline \multicolumn{1}{|c|}{ Pregunta } & \multicolumn{1}{|c|}{ Categoría } & $\begin{array}{c}\text { N. }{ }^{\circ} \text { de } \\
\text { est. }\end{array}$ & Evidencia o comentarios \\
\hline $\begin{array}{l}\text { ¿Qué has escuchado de amigos } \\
\text { familiares o vecinos acerca de ellas? }\end{array}$ & Referente a su valor estético. & 9 \\
\hline & $\begin{array}{l}\text { Asociado a su mantenimiento } \\
\text { en cautiverio. }\end{array}$ & 2 \\
\hline & Referente a sus hábitos. & 4 & Est. N. ${ }^{\circ}$ 3: Cuando calienta mucho salen artas mariposas. \\
\hline & Asociado con el peligro. & 2 & \\
\hline & & \\
\hline
\end{tabular}

En la tabla 12 se observa que algunas de las inquietudes o curiosidades iniciales expuestas en la tabla 11 de los estudiantes son más claras, sobre todo, las concernientes a la morfología y al ciclo de vida. Este puede ser el reflejo del trabajo de los estudiantes en el mariposario, ya que muchos dedicaron horas en el cuidado y seguimiento de los organismos, aun en los momentos en los que no se encontraba presente el profesor y en sus horas de descanso. 
Tabla 11. Pregunta 6 pretest

\begin{tabular}{|c|c|c|c|}
\hline Pregunta & Categoría & $\begin{array}{l}\text { N. }{ }^{\circ} \text { de } \\
\text { est. }\end{array}$ & Evidencia o comentarios \\
\hline \multirow[t]{8}{*}{ ¿Qué quisieras saber sobre ellas? } & $\begin{array}{l}\text { Referente a las etapas de sus ciclo de } \\
\text { vida y morfología. }\end{array}$ & 4 & \\
\hline & Referente a su tipo de reproducción. & 2 & \\
\hline & Referente a sus hábitos. & 3 & \\
\hline & Referente a su historia evolutiva. & 1 & Est. N. ㅇ: Cómo aparecieron las mariposas. \\
\hline & Morfología interna. & 1 & Est. N. ${ }^{\circ}$ 6: Cómo son por dentro. \\
\hline & Interacción con el ambiente. & 1 & \\
\hline & Información en general. & 3 & \\
\hline & No responde. & 2 & \\
\hline
\end{tabular}

Tabla 12. Pregunta del postest

\begin{tabular}{|c|c|c|c|}
\hline Pregunta & Categoría & $\begin{array}{l}\text { N. }{ }^{\circ} \text { de } \\
\text { est. }\end{array}$ & Evidencia o comentarios \\
\hline \multirow{5}{*}{$\begin{array}{l}\text { ¿Qué otras cosas sabes acerca de las } \\
\text { mariposas o de los insectos? }\end{array}$} & Referente a los artrópodos. & 4 & \\
\hline & $\begin{array}{l}\text { Asociado a características de } \\
\text { toxicidad o camuflaje. }\end{array}$ & 3 & Que hay unas que se camuflan \\
\hline & Referente a su morfología. & 4 & $\begin{array}{l}\text { Que hay unas mariposas que se llaman Cristal, porque } \\
\text { son trasparentes. }\end{array}$ \\
\hline & Referente a la polinización. & 5 & \\
\hline & No responde. & 1 & \\
\hline
\end{tabular}

En general, hay una ampliación en cuanto a los conocimientos de los estudiantes respecto a las mariposas, sus argumentaciones manifiestan una observación y un trabajo dedicado; además, se expresan nuevos valores, sobre todo en el manejo y la responsabilidad al trabajar con organismos vivos. También, hay nuevas apreciaciones sobre el valor de otros organismos, como los insectos en general, transformando algunas de las reacciones iniciales de reserva y miedo, a curiosidad y sorpresa por sus estructuras y papel ecológico. 
Por otra parte, esta experiencia incluyó diferentes actores de la institución como personal administrativo, personal de aseo y seguridad, profesores de otras áreas, que fueron claves para la difusión del proyecto y su éxito. Así como se muestra en la experiencia de Mendoza (2013) quien utilizó el mariposario como un recurso experiencial en el desarrollo de una cartilla llamada Preservando la biodiversidad, y en donde profesores, directores, padres de familia y personal de mantenimiento contribuyeron al desarrollo del mismo.

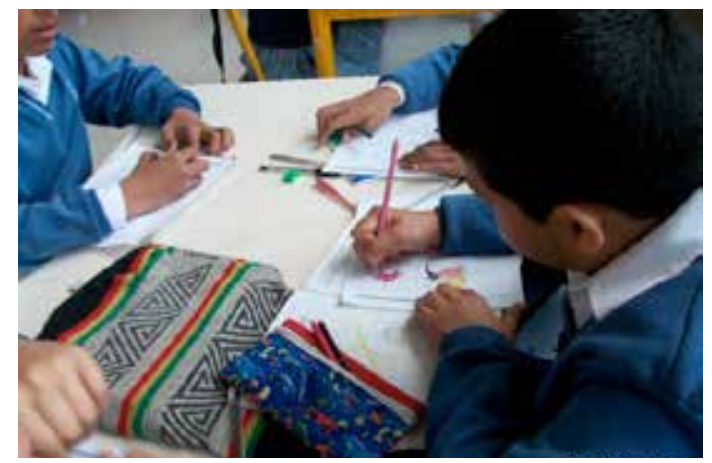

Imagen 2. Estudiantes de 702 resolviendo guía 1 Tomado por: (Gina Robayo

\section{GUÍAS UTILIZADAS.}

El trabajo alrededor de las guías demuestra ser un apoyo significativo a la hora de tratar con las mariposas y los insectos en general, ya que su prioridad no fue la de completar las mismas correctamente, si no la de brindar un espacio físico en la que los niños puedan plasmar sus propias interpretaciones, como dibujos, en su mayoría, pero también con preguntas, con actividades investigativas, etc., en las que la información fuese accesible y atractiva; y en donde alternamente ellos mismos pudieran acceder a los datos con sus palabras y con su estilo particular, para recordar o conocer nueva información.

Por ejemplo, la guía Los insectos fue una introducción a las características morfológicas de los insectos acompañada de una presentación audiovisual y de una muestra de una colección entomológica, lo que permitió que los estudiantes empezasen a reconocer lo que se considera insecto y lo que no, y por qué. Algunos estudiantes rotularon sus dibujos con sinónimos o diminutivos de las estructuras vistas como los ojos, "bolas pequeñitas con punticos guecos" (para referirse a la forma que tiene los ojos compuestos). También se observa una clara identificación de las mariposas y las mariquitas como insectos, ya que fueron las que más dibujaron, aunque prevalecen algunas características antropocéntricas.

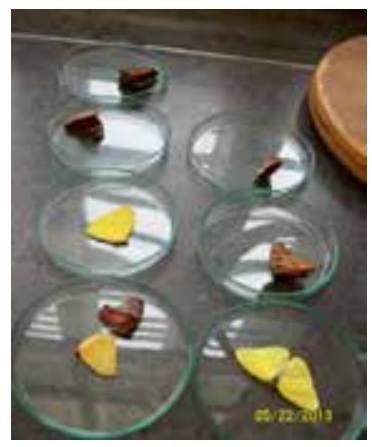

Imagen 3. Material de observación guía N. ${ }^{\circ} 2$ Tomado por: @Gina Robayo

Un aspecto importante fue el resultado del estudio de las estructuras, ya que suscitó preguntas más profundas sobre todo en cuanto al porqué algunos animales tienen estructuras repetidas (ojos y ocelos), o las formas tan variadas de las mismas en el caso de los aparatos bucales, es decir: ¿cómo los usaban?, ¿por qué los usaban?, ¿cuándo los usaban?, ¿por qué algunos no se encontraban en Colombia?, que son preguntas que ahondan en las características de los ambientes, de la relación estructura, recurso, ambiente, de la depredación, de las ventajas físicas, biológica, químicas que representan, de sus hábitos, de por qué pueden ser plagas o beneficiosas, entre otras.

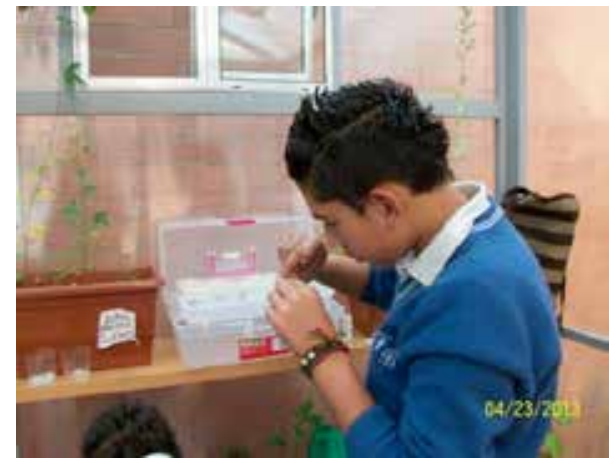

Imagen 4. Estudiante observando y marcando huevos en mariposario. Tomado por: (CGina Robayo

\section{GUÍA N. ${ }^{\circ}$ 2. LAS MARIPOSAS.}

Como resultado de las sesiones de laboratorios propuestas en las guías, se dio el primer paso hacia la construcción de la pregunta de investigación de los estudiantes. Algunos demostraron sorpresa al conocer que las alas de las mariposas están constituidas de escamas; otros, hasta no ver las escamas en el estereoscopio no se convencían, ya que la mayoría al mencionar la palabra escamas, lo relacionaron inmediatamente con la forma y el tamaño de las escamas de los peces, por lo que ver su reducido tamaño los impresionó. 
Algunos estudiantes que estuvieron en las sesiones y las visitas al mariposario, dibujaron mariposas de manera independiente en unas fichas y demostraron gran interés en conocer más.

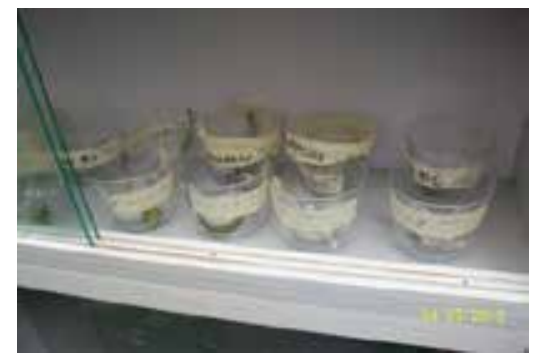

Imagen 5. Recipientes de crecimiento de orugas Tomado por: @Gina Robayo

Como lo muestran Fonseca y Medina (2009), el diseño y la aplicación de talleres basados en la EEPE permiten fortalecer las habilidades y competencias planteadas, estimulando el ejercicio de la observación y la formación de una actitud científica.

Este tipo de actividades, como los laboratorios y las observaciones, generan en el estudiante preguntas que lo ayudan a comprender los fenómenos que ve en su entorno y relacionarlos. No en vano esto dio paso a la investigación de los estudiantes.

\section{GUÍA N. 3. EL CICLO DE VIDA.}

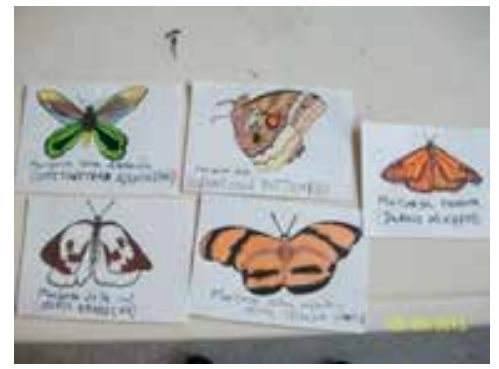

Imagen 6. Dibujos realizados por estudiantes Tomado por: (C)ina Robayo

Los estudiantes manifestaron interés por el cambio dramático que surge, en especial, en la transformación de oruga a pupa y a mariposa. Los alumnos del club tienen un mayor conocimiento de cada etapa, debido a que pasan más tiempo en el mariposario; por tanto, reconocen las diferentes estructuras concernientes a las mariposas y muestran interés por conocer acerca de las alas observadas previamente en el microscopio y estereoscopio.
Sin embargo el propósito de esta guía fue más informativo que de desarrollo, debido a que en el mariposario el estudio de la metamorfosis es más directo.

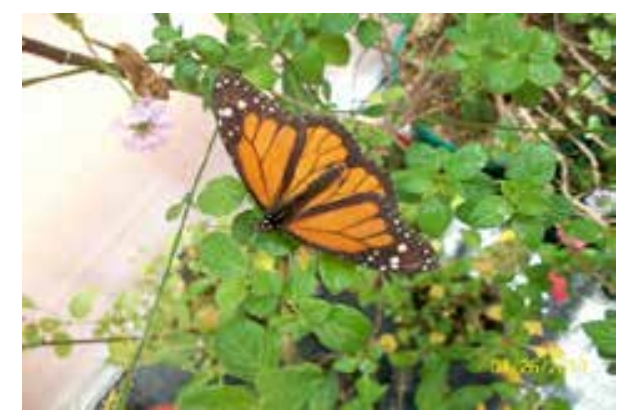

Imagen 7. Mariposa Monarca en Mariposario Panambi, Tomado por: (c) Gina Robayo

El uso de presentaciones en PowerPoint, sobre los laboratorios, y de las colecciones de insectos del club que acompañaron el trabajo de las guías, tuvieron un impacto respecto a los imaginarios de los estudiantes y en su fascinación por algunos de estos insectos. Para algunos de ellos, esta era la primera vez que veían insectos tan raros, o estructuras tan complejas como los ojos compuestos, o tan inusuales como los halterios (alas posteriores modificadas en pequeñas masas presentes en los dípteros).

\section{Ejercicio de crecimiento y desarrollo}

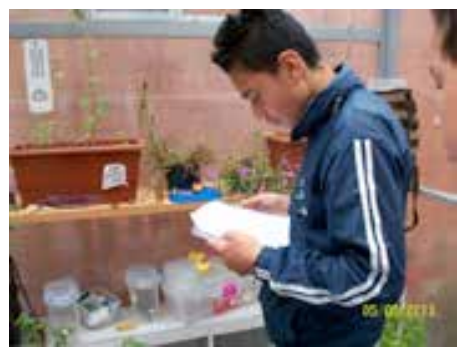

Imagen 8. Estudiante llenando tabla de seguimiento Tomado por: @Gina Robayo

Una vez se trajeron los huevos, algunos adultos y una pocas orugas, al principio los estudiantes tenían la fascinación por lo raro y literalmente querían jugar y tocar los animales; sin embargo, a medida que cada uno se responsabilizó por el cuidado y el seguimiento, empezaron a estar más pendientes del funcionamiento y de la particularidad de cada etapa de la metamorfosis. 


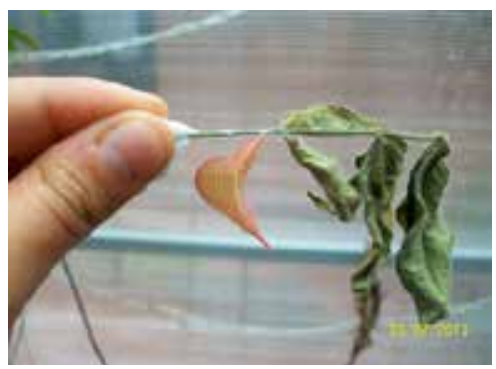

Imagen 9. Pupa de mariposa amarilla. Tomado por: @Gina Robayo

Parte del trabajo realizado en el mariposario consistía en mostrar e integrar al resto de la comunidad del colegio sobre lo que se hacía al interior del club de ciencias, por lo que los estudiantes y la profesora, recibían a la hora del descanso o a la entrada pequeños grupos de niños de otros cursos con los que se mostraban las etapas, se sembraban y regaban las plantas hospederas, además de las constantes preguntas de los niños sobre todo lo que veían. Por otro lado, se tuvo la oportunidad de mostrar parte del trabajo, a integrantes del servicio de portería y de aseo del colegio quienes, intrigados, se acercaron en varias oportunidades a escuchary a ver a los estudiantes.

El ejercicio de crecimiento y desarrollo de las mariposas fue un instrumento que no solo se relacionó con la observación del ciclo de vida de unos organismos, sino que permitió la integración de una comunidad educativa que sentía curiosidad y aprecio por las mariposas. De ahí que en el proyecto estuvieron relacionados desde el rector del colegio -quien cabe mencionar fue el que tuvo la iniciativa del mariposario-, vigilantes, colaboradores del aseo, profesores, cada uno aportando desde su conocimiento para el mantenimiento y desarrollo del mariposario.

Por otro lado, la experiencia permitió que los estudiantes desarrollaran habilidades y conocimientos a la hora de realizar prácticas similares, como la observación por medio de tablas de seguimiento, que fueron plateadas por ellos mismos; además, de tener en cuenta las condiciones éticas y físicas en el mantenimiento de organismos en lugares como el mariposario, ya que, los estudiantes estuvieron directamente involucrados en aprender y buscar cómo mantener las condiciones óptimas. Esto muestra una transición de las apreciaciones de los estudiantes frente a los organismos en general, ya que al inicio las mariposas y el mariposario fueron vistos más como atracción que como un lugar de aprendizaje y de trabajo, equivalente al realizado en el salón de clases. Lo que puede más tarde aplicarse a otros espacios académicos con otros organismos.

\section{PROYECTO DE LOS ESTUDIANTES.}

Parte del trabajo realizado en el mariposario consistió en la construcción de un proyecto de investigación inspirado en el trabajo en el mariposario que fuera realizado por los estudiantes.

Como resultado, los alumnos plantearon la siguiente pregunta: "¿El patrón de la forma, la organización y ubicación de las escamas de color negro de las mariposas Monarca pueden servir para diseñar paneles solares que sean más eficientes?".

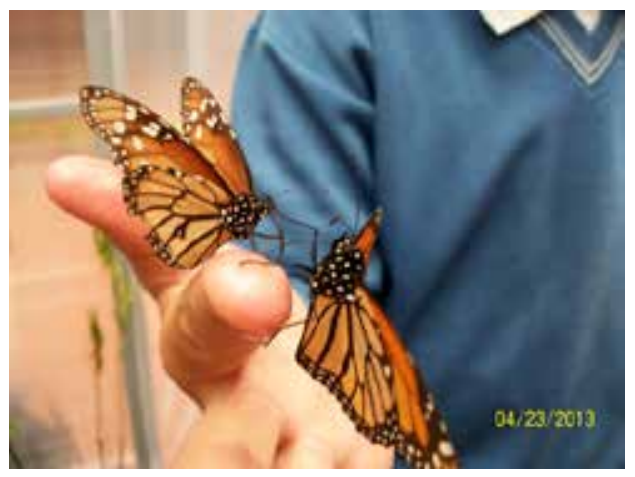

Imagen 10. Estudiante alimentando Mariposas Monarca. Tomado por: (CGina Robayo.

Esta pregunta surgió debido a que en una sesión del club, mientras se discutía sobre tecnologías inspiradas en la naturaleza, el profesor a cargo comentó un artículo en el que investigadores estaban estudiando las escamas de las mariposas debido a su potencial para el diseño de paneles solares más eficientes. De allí que los estudiantes quisieron intentar diseñar su propio panel a partir de las mariposas del mariposario, específicamente las Monarca (Danaus plexippus).

Por otra parte, gracias a que una cifra importante de las investigaciones realizadas en el club están relacionadas con el cambio climático y sus efectos, muchos de los proyectos realizados están dirigidos a crear tecnologías que no contribuyan a incrementar los efectos del cambio climático.

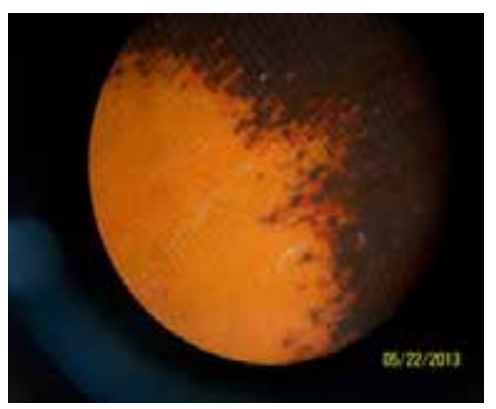

Imagen 11. Escamas de Monarca. Tomado por: @Gina Robayo 


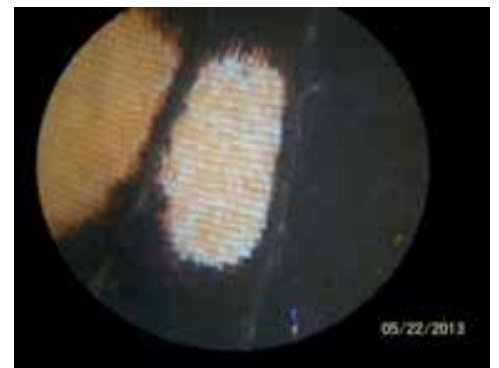

Imagen 12.Escamas de mariposa al estéreo. Tomado por: (c)ina Robayo

Los estudiantes empezaron a observar regiones del ala de la mariposa Monarca, específicamente las regiones del ala de color negro, buscando en primer lugar reconocer la forma de las escamas, si hay formas y tamaños diferentes, y si su ubicación y organización varía o no según la región del ala que se observe. En las observaciones iniciales, los estudiantes notaron que el ancho de las mismas varía según el lugar en el que se encuentran y con ello las formas y tañamos también lo hacen. De manera alternativa, los alumnos han estado informándose del funcionamiento de los paneles solares con los que plantearon un laboratorio para la fabricación de celdas solares y paneles en la institución. Sin embargo, el proyecto de los estudiantes de la línea aún está en proceso, debido a que no se ha realizado un diseño ni se ha empezado la fabricación, aunque se espera que esto se ejecute en el tiempo que resta del año lectivo.

Lo anterior demuestra que en espacios pequeños, comunes y con los objetivos y las motivaciones, se puede investigar y hacer ciencia en los colegios. Parte de ello tiene que ver con la posibilidad que se brinda para realizar proyectos como este. Muchas veces se tiene la imagen de la investigación compleja, costosa y larga que restringe en el estudiante y algunas veces en el profesor la intensión de investigar.

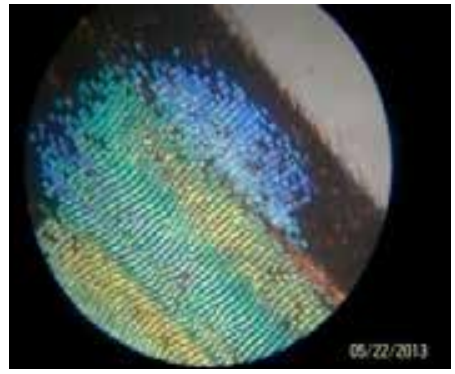

Imagen 13. Escamas de Monarca al estéreo. Tomado por: (Gina Robayo
La realización de ese proyecto muestra cómo desde un trabajo sencillo, como es el crecimiento y desarrollo de una mariposa en el mariposario puede surgir la idea de un proyecto con un potencial grandísimo de completarse exitosamente. Una fracción de ello surge de proporcionar un espacio (mariposario, club), y unas discusiones, respecto a la cotidianidad de los mismos estudiantes, de sus intereses y necesidades, que le dé significado y objetivos al trabajo realizado. Sin ello la investigación se vuelve una carga o un trabajo sin mayor trascendencia.

\section{La Entrevista}

\section{Respuesta $N^{\circ} 1$.}

Existe un reconocimiento por parte de la comunidad educativa sobre el trabajo que se ha desarrollado, tanto así, que la intensificación del trabajo ahí, trayendo las especies, ha contribuido de una u otra forma también a que sea un punto de atracción para muchas personas que desconocen completamente cuál es el ciclo de vida de una mariposa, obviamente los niños, pero también, profesores, señoras del aseo, celadores, y eso pues a mí me ha parecido clave porque prácticamente más allá de hablar de mariposas, también, está el hecho de tener una actitud frente a los seres vivos, un poco más de solidaridad con los seres vivos, que es lo que a nosotros nos hace falta muchísimo en los espacios digámoslo así en la ciudad.

Se observa un reconocimiento de la importancia y de las posibilidades que ofrece un lugar como el mariposario; se recalca el impacto que tiene en la comunidad educativa, que es un punto a favor muy significativo, ya que involucra diferentes formas de pensar, de sentir y de ver, que concurren en las instituciones y que pueden representar una ventaja a la hora de implementar diferentes proyectos en los colegios. Además, resalta el hecho de que espacios como el mariposario pueden proporcionar visones más sensibilizadas y reflexivas de la acción del hombre sobre sí mismo, y sobre su ambiente. Como lo menciona Torres (2004), los mariposarios cumplen una función importante en la sensibilidad por lo natural, moldeando la conducta relacionada con la apreciación y utilización de la naturaleza en la personas.

\section{Respuesta $N^{\circ} 2$.}

[...] en principio se nota la preparación, la coherencia que tiene el discurso con la preparación y con las expectativas de los muchachos y es importante también recalcar que si bien el proceso ha sido digámoslo así, muy rápido, por que es practica integral [...]. 
[...] La intensificación en estas horas nos ha permitido, de una u otra forma, reconocer el potencial que tienes tú en muchos aspectos, que no son solamente de la vida académica, sino también de la vida de ser humano, y es muy importante que uno lo rescate, y esa es también la filosofía del proyecto curricular, que antes que uno hablar de licenciados uno hable de visión humana, rescate toda esa serie de potencialidades, características [...].

[...] Antes que nada, estamos trabajando con muchachos de carne y hueso que necesitan una orientación en unos aspectos que uno cree que ya fueron superados durante etapas tempranas, pero que en realidad se ven muchas falencias y el punto no es criticar a quienes no hicieron, sino hacer algo desde ya.

Por otra parte se muestra la necesidad de idear nuevas formas de llegar al estudiante que tangan mayor impacto y que estén en concordancia con la expectativas tanto del profesor como del estudiante, que sean coherentes con el discurso y con la práctica, de allí que el mariposario no solo es visto como un potencial académico, sino también humano que exige respeto, responsabilidad, compromiso y disciplina. Fonseca y Medina (2009) lo mencionan como espacios externos al aula tradicional de clase que permiten enseñar y aprender a través del hacer, que generan expectativas, curiosidad y motivación por aprender, ya que permiten establecer relaciones entre diferentes conceptos mediante la práctica y experiencia.

\section{Conclusión}

Los mariposarios son espacios de observación, exploración, experimentación e investigación que involucran no solo aspectos investigativos sino también humanos, que ofrecen un lugar alterno e interactivo que intenta atraer o seducir al estudiante y mantenerlo con una serie de posibilidades que están sujetas a la imaginación y a las expectativas de quien lo desarrolle; además puede abarcar no solo al estudiante sino también gran parte de su núcleo familiar y educativo, lo que enriquece y contextualiza mucho más la experiencia de aprender y de enseñar.

Este tipo de espacios se convierten en instrumentos valiosos para el licenciado en biología y también para la enseñanza de las ciencias en general, ya que atraen al estudiante y le permiten al profesor trabajar desde diferentes aspecto pasando desde lo puro estético, hasta lo técnico o reflexivo. Y que pueden ser un punto más accesible y más significativo, de manera que el estudiante no solo lo aprecie, sino que lo apropie.
La práctica pedagógica permite un acercamiento a los contextos educativos que le brindan al futuro licenciado en biología la experiencia de compartir y de vivir el quehacer del maestro, enriqueciendo su identidad como profesor, como estudiante y como persona, enfrentándolo a un aula de clase, a un grupo de personas y a una institución. Yllevándolo a reconocer sus fortalezas, pero también sus aspectos a mejorar que contribuyan a su formación como licenciado en Biología consciente del contexto educativo en el que se desarrolla su práctica, y de la sociedad de la que forma parte.

\section{Agradecimientos}

A las directivas y comunidad académica del Colegio Orlando Fals Borda IDE, por permitirme realizar este proyecto en sus instalaciones, por su apoyo y contribuciones al llevar a cabo las diferentes actividades, por su constante interés, compromiso y dedicación con el proyecto del mariposario.

Al profesor Yair Porras, por sus constantes enseñanzas, por su confianza al permitirme realizar el proyecto en el colegio y en su aula de clase. Por su ejemplo de maestro y por su incondicional ayuda, comprensión y apoyo, sin el cual no habría podido realizar la práctica.

A los estudiantes y profesores del club de ciencias Tataxue, por recibirme con los brazos abiertos y permitirme ser parte de su familia. Por todo el entusiasmo y disposición al realizar las actividades y por todo lo que aprendí de ustedes. También en agradecimiento de la profesora Ivonne Angarita, por su ejemplo y confianza al recibirme y también por sus enseñanzas.

A los estudiantes de los grados $801,802,702$ y sexto, por permitirme estar en sus clases, por su alegría, por su paciencia y porque enriquecieron mi práctica y mi ser maestro.

Al rector, los profesores, vigilantes, colaboradores del aseo y bibliotecarios, por su colaboración incondicional, sus concejos y aportes los cuales me ayudaron e hicieron del proyecto lo que es.

A la línea de investigación de faunística y conservación de artrópodos y a la profesora Sonia Martínez por su orientación, paciencia y concejos en la dirección del proyecto. 


\section{Referencias Bibliográficas}

Arango, M.; Chaves, M. y Feinsinger, P. (2002). Guía metodológica para la Enseñanza de la Ecología en el Patio de la Escuela. Nueva York: National Audubon Society.

Bonilla, E. y Rodríguez, P. (1997). Más allá del dilema de los métodos: La investigación en ciencias sociales. $3 a$. ed. Bogotá: Grupo Editorial Norma.

Borror, D. y White, R. (1970). A field guide to insects. Boston: Petersons Field Guides.

Colegio Orlando Fals Borda IED (2010). Proyecto Educativo Institucional PEI. Recuperado el 7 de diciembre de 2012 de: http://www.colegioorlandofalsborda.edu.co/Documentos.html

Colegio Orlando Fals Borda IED (2012). Ciudadanos ante el cambio climático: Una mirada de la realidad ambiental desde la perspectiva astronómica del Club de Astronomía y Ciencias Tataxue. Recuperado el 7 de diciembre de 2012 de: http:// www.google.com.co/url?sa=t\&rct=j\&q=\&esrc $=s \&-$ frm $=1 \&$ source $=$ web\&cd $=3 \& v e d=0 C C g Q F j A C \& u r-$ l=http $\% 3 \mathrm{~A} \% 2 \mathrm{~F} \% 2 \mathrm{Fwww}$.colegioorlandofalsborda. edu.co\%2FArchivos_pdf\%2FPRO_TATAXUE_2012. pdf\&ei=tZpiVOXrNoKXNueYgbAF\&usg=AFQjCNEuLLMuc3dFBoiOydPeoARqTuoLaQ\&sig2=IoiYKCVVe23W3ESvrl20uA

Colegio Orlando Fals Borda IED (2012). En red-andote con el ambiente, hacia la formación de una ciudadanía ambientalmente responsable del Colegio Orlando Fals Borda. Recuperado el 7 de diciembre de 2012 de: http://www.colegioorlandofalsborda.edu.co/ Proyectos_Transversales.html

Fonseca, Y. y Medina, D. (2009). El mariposario del departamento de Biología como estrategia para la enseñanza de las ciencias naturales en la básica primaria. Tesis de pregrado no publicada. Bogotá: Universidad Pedagógica Nacional.

Fundación Sirua y Fauna y Flora Internacional (2006). Centro de manejo de vida silvestre Awacachi. Recuperado el 2 de septiembre de 2012 de: http://www.google.com.co/url?sa=$t \& r c t=j \& q=\& e s r c=s \& f r m=1 \&$ source $=w e b \& c d=2 \&$ ved $=0 C C Q Q F j A B \& u r l=h t t p \% 3 A \% 2 F \% 2 F w w w$. darwininitiative.org.uk\%2Fdocuments\%2F13005\%2F3177\%2F13005\%2520FR\%2520App11\%2520Management\%2520Plan.pdf\&ei=BI1iVKPDF8yggwToh4DgCg\&usg=AFQjCNFPheSPZT1INywB0SXLK1Aron5KrA\&sig2=OYzG6mXr2MZg8_g7cuFYTA
Gullan, P. y Cranston, P. (2010). The Insects: An Outline of Entomology. 4a., Oxford, Reino Unido. Editorial. Wiley - Blackwell.

Martínez, M. (s.f.). El enfoque cualitativo de la investigación. Recuperado el 15 de septiembre de 2012 de: http://prof.usb.ve/miguelm/nc10enfoquecualitativo.html

Mendoza, X. (2013). Mariposario alitas multicolores: Alfabetización ambiental asociada a la pérdida de especies. Tesis de pregrado no publicada. Bogotá: Universidad Pedagógica Nacional.

Torres, R. (2004). Mariposas para educar. Bogotá: Universidad Pedagógica Nacional. 